\title{
Remimazolam besilate, a benzodiazepine, has been approved for general anesthesia!!
}

\author{
Kenichi Masui ${ }^{1}$ \\ Received: 15 February 2020 / Accepted: 22 February 2020 / Published online: 10 March 2020 \\ (c) Japanese Society of Anesthesiologists 2020
}

Keywords Remimazolam $\cdot$ General anesthetic $\cdot$ Benzodiazepine

\section{Introduction: consideration of advantages}

In January 2020, remimazolam besilate, a novel benzodiazepine, was approved as a general anesthetic in Japan firstly in the world. A novel anesthetic, remimazolam, would be desired to have advantages beyond existing anesthetics such as inhalation anesthetics, propofol, and midazolam. Inhalation anesthetics might trigger malignant hyperthermia, and is a risk for postoperative nausea and vomiting (PONV), while a benzodiazepine, midazolam, is not a trigger for malignant hyperthermia and has an advantage to reduce PONV [1]. Remimazolam induced mild and transient nausea after bolus but not seems to have high risk of PONV [2]. An inhalation anesthetic but not an intravenous anesthetic including remimazolam pollutes environmental air including in the operation room [3]. Large dose and longer infusion of propofol is a risk of propofol infusion syndrome in both children and adults [4]. Injection pain of propofol is also a clear disadvantage although the use of lidocaine or opioid may reduce the injection pain [5, 6]. Remimazolam had no notable injection pain in a phase I clinical trial [2]. Midazolam is sometimes used for general anesthesia but not often, maybe due to long-acting property than propofol and inhalation anesthetics and large interindividual variability [7]. Prolonged effect of an active metabolite of midazolam may cause a difficulty to use midazolam [8], while remimazolam is metabolized by tissue esterases to an inactive metabolite [9]. Context-sensitive half-time (CSHT) estimated from a pharmacokinetic/pharmacodynamic analysis has revealed that the off-set of the remimazolam after the

Kenichi Masui

kenichi@masuinet.com

1 Department of Anesthesiology, Showa University School of Medicine, Hatanodai 1-5-8, Shinagawa, Tokyo 142-8666, Japan stop of its infusion is faster than that of the midazolam. For instance, when comparing half-time after $3 \mathrm{~h}$ constant rate infusion, the half-time of remimazolam is approximately one-fifth shorter than that of midazolam [10]. Note that the researchers calculated CSHT in untypical way (the detail is discussed below). On the above-mentioned beneficial properties, remimazolam has a potential to be a principal intravenous anesthetic for general anesthesia.

\section{How rapid of the offset?}

Remimazolam may be recognized to be rapid offset or ultrashort acting drug, but how rapid? When seeing CSHT in an article again, the half-time of arterial remimazolam concentration for $3 \mathrm{~h}$ constant rate infusion is approximately $7.5 \mathrm{~min}$ [10]. In the original article, CSHT is calculated using target-controlled infusion [11]. The half-time for $3 \mathrm{~h}$ $\mathrm{TCI}$ is longer than the half-time for $3 \mathrm{~h}$ constant rate infusion [12]. When seeing propofol CSHT, the half-time for $3 \mathrm{~h} \mathrm{TCI}$ using Marsh model is $8.6 \mathrm{~min}$, while the half-time for $3 \mathrm{~h}$ constant rate infusion is $7.5 \mathrm{~min}$. Both half-times after $3 \mathrm{~h}$ constant rate infusion are $7.5 \mathrm{~min}$, suggesting that remimazolam and propofol may have similar concentration decay curve after stop of continuous infusion. In other words, remimazolam may have rapid offset profile similar to propofol. However, in the study showing remimazolam CSHT [10], remimazolam was infused over just $1 \mathrm{~min}$. Another pharmacokinetic model of remimazolam developed using dataset obtained during general anesthesia might result in another CSHT profile. 


\section{Electroencephalographic index}

Assessment of the effect of remimazolam during anesthesia would be practical issue especially for the next years after the start of the distribution. Nowadays, we often use electroencephalographic (EEG) monitor to assess the effect of anesthetics, especially for intravenous anesthetic due to larger interindividual variability than inhalation anesthetic. Appropriate ranges of EEG indices for remimazolam anesthesia are unclear, the ranges may be higher, e.g., 60-70 of BIS index or 50-60 of patient state index [2]. The databases to develop the EEG indices do not include EEG data from patients under remimazolam anesthesia, currently. These EEG indices or alternative EEG derived values such as spectral edge frequency 95 would be expected to determine appropriate level of remimazolam for general anesthesia.

\section{Pharmacokinetic/pharmacodynamic model for predicted concentration}

To have remimazolam concentration to control anesthesia level, pharmacokinetic and pharmacodynamic models are useful. Although a pharmacokinetic model with a pharmacodynamic model has been published [10], the final pharmacokinetic model is not a compartment model but a recirculatory model. A compartment model of remimazolam for general anesthesia is desired with following reasons: (1) clinically available medical devices showing the predicted drug concentration use compartment models, (2) the external validity of the published pharmacokinetic model is unknown [13] for general anesthesia patients, because the pharmacokinetic model was developed using the dataset derived from patients who received only 1 min infusion of remimazolam. Population pharmacokinetic and pharmacodynamic models will help to make and assess dose regimens in clinical practice $[14,15]$.

\section{Body weight for dose regimen}

Body weight is generally considered to determine bolus dose or infusion rate of a drug. For remimazolam, a previous study concluded that "dosing by body weight may offer no advantage over fixed doses in terms of consistency of exposure to remimazolam within the weight range studied (65-90 kg)." [10] Here is issues whether body weight has no impact on pharmacokinetic profile on remimazolam. Rapid infusion of remimazolam like bolus may cause large variability of remimazolam concentration time course [16]. Large concentration variability may conceal the influence of a covariate such as body weight on pharmacokinetic profile and pharmacokinetic parameters. On the other hand, there is a study suggesting little impact of body weight on plasma concentration of a drug, where a pharmacokinetic model excluding body weight as a covariate predicts plasma concentration better than other pharmacokinetic models which incorporate body weight as a covariate [17]. In thin and obese patients, body weight may have an impact on remimazolam concentration $[18,19]$. The influence of body weight should be examined using the dataset derived from patients who receive continuous infusion of remimazolam for general anesthesia.

\section{Hypotension: side effect of induction agent}

Hypotension is frequently observed as a side effect during induction of anesthesia using propofol [20-22]. A phase I clinical trial for remimazolam revealed that a 1-min bolus infusion of remimazolam $0.01-0.30 \mathrm{mg} / \mathrm{kg}$ did not cause hypotension, which was defined as systolic blood pressure lower than $80 \mathrm{mmHg}$, except one occurrence of hypotension $8 \mathrm{~h}$ after the dose of remimazolam [2]. Although this clinical trial for remimazolam was performed in healthy subject, a current induction agent, propofol can cause hypotension at concentrations during induction in healthy subjects [23]. There may be less occurrence of hypotension by remimazolam than propofol in healthy patients. There is no published data for hypotension in poor risk patients for remimazolam.

\section{Influence of hepatic and renal impairment}

How is the pharmacokinetic alteration of remimazolam in patients with hepatic or renal impairment? Remimazolam is expected to have organ-independent elimination as the following pharmacokinetic profile [24]: (1) incorporation of carboxylic ester, which is metabolized by tissue esterase, (2) 300-times lower affinity of metabolized compound (CNS 7054, which is carboxylic acid) than remimazolam (CNS 7056) [2], and (3) full agonist. This is the translated concept from remifentanil [24]. Accumulation is related issue for a large total dose or a long high-dose infusion of remimazolam. As the terminal phase half-life of the main metabolite, CNS 7054, is 3-5 times longer than that of remimazolam [2], CNS 7054 can accumulate. Although this metabolites 300-times lower affinity than remimazolam, large accumulation might cause sedation especially in patients with hepatic or renal impairment. Clinical studies are necessary in these specific situations. 


\section{Pediatric anesthesia}

For total intravenous anesthesia in pediatric patients [25], remimazolam might be preferable because of less injection pain and no risk of propofol infusion syndrome. Propofol has an advantage to reduce laryngospasm and airway reactivity [26] but midazolam rarely cause laryngospasm [27]. The risk of emergence laryngospasm by remimazolam might be low due to rapid elimination profile. Survey of remimazolam anesthesia in pediatric patients to clarify the safety and efficacy is desired in the near future.

\section{Procedural sedation}

For procedural sedation in emergency department in Japan, thiopental and midazolam are frequently used [28]. As these drugs have large pharmacodynamic variability and long CSHT after a long infusion [11], remimazolam, which has shorter CSHT, would be preferable. In some countries, clinical trials for approval have been implemented [2, 10, 23]. Currently, procedural sedation with remimazolam is offlabel in Japan, but the approval for that is expected.

\section{Publications}

Several animals studies [29-32] and clinical trials for the phase I to III studies [2, 10, 23, 33, 34] have been published so that we can access useful information for remimazolam. As published human studies are limited in volunteers and procedural sedated patients, clinical studies for remimazolam anesthesia are necessary.

\section{Conclusion}

A novel benzodiazepine, remimazolam, has been approved as a general anesthetic. Remimazolam was developed as a soft drug, which is designed to be active, easily transformed to inactive metabolites. Future clinical experiences and experiments will clarify the utility and profile.

\section{Compliance with ethical standards}

Conflict of interest Kenichi Masui, MD, PhD. wrote and approved the final manuscript. He is a consultant/advisor for, and has a research grant from Mundipharma K.K. He has had no involvement with the human studies of remimazolam.

\section{References}

1. Gan TJ, Diemunsch P, Habib AS, Kovac A, Kranke P, Meyer TA, Watcha M, Chung F, Angus S, Apfel CC, Bergese SD, Candiotti KA, Chan MT, Davis PJ, Hooper VD, Lagoo-Deenadayalan S, Myles P, Nezat G, Philip BK, Tramer MR, Society for ambulatory A. Consensus guidelines for the management of postoperative nausea and vomiting. Anesth Analg. 2014;118:85-113.

2. Antonik LJ, Goldwater DR, Kilpatrick GJ, Tilbrook GS, Borkett KM. A placebo- and midazolam-controlled phase I single ascending-dose study evaluating the safety, pharmacokinetics, and pharmacodynamics of remimazolam (CNS 7056): part I. Safety, efficacy, and basic pharmacokinetics. Anesth Analg. 2012;115:274-83.

3. Deng H-B, Li F-X, Cai Y-H, Xu S-Y. Waste anesthetic gas exposure and strategies for solution. J Anesth. 2018;32:269-82.

4. Hemphill S, McMenamin L, Bellamy MC, Hopkins PM. Propofol infusion syndrome: a structured literature review and analysis of published case reports. Br J Anaesth. 2019;122:448-59.

5. Basaranoglu G, Erden V, Delatioglu H, Saitoglu L. Reduction of pain on injection of propofol using meperidine and remifentanil. Eur J Anaesthesiol. 2005;22:890-2.

6. Li X, Chen C-j, Tan F, Pan J-r, Xing J-b, Zhu Q-q, Hei Z-q, Zhou S-1. Effect of dexmedetomidine for attenuation of propofol injection pain in electroconvulsive therapy: a randomized controlled study. J Anesth. 2018;32:70-6.

7. Franken LG, de Winter BCM, Masman AD, van Dijk M, Baar FPM, Tibboel D, Koch BCP, van Gelder T, Mathot RAA. Population pharmacodynamic modelling of midazolam induced sedation in terminally ill adult patients. Br J Clin Pharmacol. 2018;84:320-30.

8. Bauer TM, Ritz R, Haberthür C, Haefeli WE, Scollo-Lavizzari G, Ha HR, Hunkeler W, Sleight AJ. Prolonged sedation due to accumulation of conjugated metabolites of midazolam. Lancet. 1995;346:145-7.

9. Sneyd JR. Remimazolam: new beginnings or just a me-too? Anesth Analg. 2012;115:217-9.

10. Wiltshire HR, Kilpatrick GJ, Tilbrook GS, Borkett KM. A placebo- and midazolam-controlled phase I single ascending-dose study evaluating the safety, pharmacokinetics, and pharmacodynamics of remimazolam (CNS 7056): Part II. Population pharmacokinetic and pharmacodynamic modeling and simulation. Anesth Analg. 2012;115:284-96.

11. Hughes MA, Glass PS, Jacobs JR. Context-sensitive half-time in multicompartment pharmacokinetic models for intravenous anesthetic drugs. Anesthesiology. 1992;76:334-41.

12. Schnider T, Minto C. Context sensitive decrement times of remimazolam. Anesth Analg. 2013;117:285.

13. Obara S, Imaizumi T, Hakozaki T, Hosono A, Iseki Y, Sanbe N, Murakawa M. Evaluation of pharmacokinetic models of intravenous dexmedetomidine in sedated patients under spinal anesthesia. J Anesth. 2018;32:33-40.

14. Lee S, Kim I, Pyeon T, Lee S, Song J, Rhee J, Jeong S. Population pharmacokinetics of palonosetron and model-based assessment of dosing strategies. J Anesth. 2019;33:381-9.

15. Masui K, Ishigaki S, Tomita A, Otake H. Rocuronium pharmacodynamic models for published five pharmacokinetic models: age and sex are covariates in pharmacodynamic models. J Anesth. 2018:32:709-16.

16. Masui K, Kira M, Kazama T, Hagihira S, Mortier EP, Struys MM. Early phase pharmacokinetics but not pharmacodynamics are influenced by propofol infusion rate. Anesthesiology. 2009;111:805-17.

17. Sasakawa T, Masui K, Kazama T, Iwasaki H. The predictive ability of six pharmacokinetic models of rocuronium developed using 
a single bolus: evaluation with bolus and continuous infusion regimen. J Anesth. 2016;30:620-7.

18. Park JH, Choi SM, Park JH, Lee KH, Yun HJ, Lee EK, Choi BM, Noh GJ. Population pharmacokinetic analysis of propofol in underweight patients under general anaesthesia. $\mathrm{Br} \mathrm{J}$ Anaesth. 2018;121:559-66.

19. Cortinez LI, De la Fuente N, Eleveld DJ, Oliveros A, Crovari F, Sepulveda P, Ibacache M, Solari S. Performance of propofol target-controlled infusion models in the obese: pharmacokinetic and pharmacodynamic analysis. Anesth Analg. 2014;119:302-10.

20. Claeys MA, Gepts E, Camu F. Haemodynamic changes during anaesthesia induced and maintained with propofol. Br J Anaesth. 1988;60:3-9.

21. Hino H, Matsuura T, Kihara Y, Tsujikawa S, Mori T, Nishikawa $\mathrm{K}$. Comparison between hemodynamic effects of propofol and thiopental during general anesthesia induction with remifentanil infusion: a double-blind, age-stratified, randomized study. J Anesth. 2019;33:509-15.

22. Jor O, Maca J, Koutna J, Gemrotova M, Vymazal T, Litschmannova M, Sevcik P, Reimer P, Mikulova V, Trlicova M, Cerny V. Hypotension after induction of general anesthesia: occurrence, risk factors, and therapy. A prospective multicentre observational study. J Anesth. 2018;32:673-80.

23. Borkett KM, Riff DS, Schwartz HI, Winkle PJ, Pambianco DJ, Lees JP, Wilhelm-Ogunbiyi K. A phase iia, randomized, double-blind study of remimazolam (CNS 7056) versus midazolam for sedation in upper gastrointestinal endoscopy. Anesth Analg. 2015;120:771-80.

24. Stafford JA, Pacofsky GJ, Cox RF, Cowan JR, Dorsey GF, Gonzales SS, Jung DK, Koszalka GW, McIntyre MS, Tidwell JH, Wiard RP, Feldman PL. Identification and structure-activity studies of novel ultrashort-acting benzodiazepine receptor agonists. Bioorg Med Chem Lett. 2002;12:3215-8.

25. Biricik E, Karacaer F, Güleç E, Sürmelioğlu Ö, Ilgınel M, Özcengiz D. Comparison of TIVA with different combinations of ketamine-propofol mixtures in pediatric patients. J Anesth. 2018;32:104-11.

26. Gaynor J, Ansermino JM. Paediatric total intravenous anaesthesia. BJA. Education. 2016;16:369-73.
27. Davis DP, Hamilton RS, Webster TH. Reversal of midazolaminduced laryngospasm with flumazenil. Ann Emerg Med. 1998;32:263-5.

28. Norii T, Homma Y, Shimizu H, Takase H, Kim S-H, Nagata S, Shimosato A, Crandall C, Norii T, Takase H, Homma Y, Shimizu H, Kim S-H, Nagata S, Noma T, Fukano K, Shimosato A, Sedation ObotJP, investigators AR. Procedural sedation and analgesia in the emergency department in Japan: interim analysis of multicenter prospective observational study. J Anesth. 2019;33:238-49.

29. Kilpatrick GJ, McIntyre MS, Cox RF, Stafford JA, Pacofsky GJ, Lovell GG, Wiard RP, Feldman PL, Collins H, Waszczak BL, Tilbrook GS. CNS 7056: a novel ultra-short-acting Benzodiazepine. Anesthesiology. 2007;107:60-6.

30. Upton R, Martinez A, Grant C. A dose escalation study in sheep of the effects of the benzodiazepine CNS 7056 on sedation, the EEG and the respiratory and cardiovascular systems. Br J Pharmacol. 2008;155:52-61.

31. Upton RN, Martinez AM, Grant C. Comparison of the sedative properties of CNS 7056, midazolam, and propofol in sheep. Br J Anaesth. 2009;103:848-57.

32. Upton RN, Somogyi AA, Martinez AM, Colvill J, Grant C. Pharmacokinetics and pharmacodynamics of the short-acting sedative CNS 7056 in sheep. Br J Anaesth. 2010;105:798-809.

33. Worthington MT, Antonik LJ, Goldwater DR, Lees JP, WilhelmOgunbiyi K, Borkett KM, Mitchell MC. A phase Ib, dose-finding study of multiple doses of remimazolam (CNS 7056) in volunteers undergoing colonoscopy. Anesth Analg. 2013;117:1093-100.

34. Rex DK, Bhandari R, Desta T, DeMicco MP, Schaeffer C, Etzkorn K, Barish CF, Pruitt R, Cash BD, Quirk D, Tiongco F, Sullivan S, Bernstein D. A phase III study evaluating the efficacy and safety of remimazolam (CNS 7056) compared with placebo and midazolam in patients undergoing colonoscopy. Gastrointest Endosc. 2018;88(427-37):e6.

Publisher's Note Springer Nature remains neutral with regard to jurisdictional claims in published maps and institutional affiliations. 